This document is confidential and is proprietary to the American Chemical Society and its authors. Do not copy or disclose without written permission. If you have received this item in error, notify the sender and delete all copies.

\title{
Hydrogenation of Graphene by Reaction at High Pressure and High Temperature
}

\begin{tabular}{|c|c|}
\hline Journal: & ACS Nano \\
\hline Manuscript ID: & Draft \\
\hline Manuscript Type: & Article \\
\hline Date Submitted by the Author: & $\mathrm{n} / \mathrm{a}$ \\
\hline Complete List of Authors: & $\begin{array}{l}\text { Smith, Dean; University of Salford, School of Computing, Science \& } \\
\text { Engineering } \\
\text { Howie, Ross; The University of Edinburgh, Centre for Science at Extreme } \\
\text { Conditions and School of Physics and Astronomy } \\
\text { Crowe, Iain; University of Manchester, Photon Science Institute and School } \\
\text { of Electrical and Electronic Engineering } \\
\text { Simionescu, Cristina; University of Salford, School of Computing, Science \& } \\
\text { Engineering } \\
\text { Muryn, Christopher; University of Manchester, Photon Science Institute and } \\
\text { School of Chemistry } \\
\text { Vishnyakov, Vladimir; University of Huddersfield, School of Computing and } \\
\text { Engineering } \\
\text { Novoselov, Konstantin; University of Manchester, School of Physics and } \\
\text { Astronomy } \\
\text { Kim, Yong-Jin; University of Manchester, School of Physics and Astronomy } \\
\text { Halsall, Matthew; University of Manchester, Photon Science Institute and } \\
\text { School of Electrical and Electronic Engineering } \\
\text { Gregoryanz, Eugene; The University of Edinburgh, Centre for Science at } \\
\text { Extreme Conditions and School of Physics and Astronomy } \\
\text { Proctor, John; University of Salford, School of Computing, Science \& } \\
\text { Engineering }\end{array}$ \\
\hline
\end{tabular}

\section{SCHOLARONE ${ }^{\text {m }}$ \\ Manuscripts}




\title{
Hydrogenation of Graphene by Reaction at High
}

\section{Pressure and High Temperature}

\author{
Dean Smith, ${ }^{\dagger}, *$ Ross T. Howie,,${ }^{\ddagger}$ Iain F. Crowe ${ }^{\S}$ Cristina L. Simionescu, ${ }^{\dagger}$ Chris Muryn, ${ }^{\perp}$ \\ Vladimir Vishnyakov," Konstantin S. Novoselov, "Yong-Jin Kim, " Matthew P. Halsall, ${ }^{\S}$ Eugene \\ Gregoryanz, ${ }^{\ddagger} \&$ John E. Proctor ${ }^{\dagger, \S, * *}$ \\ ${ }^{\dagger}$ School of Computing, Science \& Engineering, University of Salford, Salford, M5 4WT, UK \\ Centre for Science at Extreme Conditions and School of Physics and Astronomy, The \\ University of Edinburgh, Edinburgh, EH9 3JZ, UK \\ §Photon Science Institute and School of Electrical and Electronic Engineering, University of \\ Manchester, Manchester, M13 9PL, UK \\ ${ }^{\perp}$ Photon Science Institute and School of Chemistry, University of Manchester, Manchester M13 \\ 9PL, UK

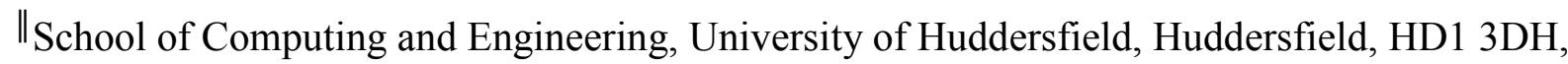 \\ UK \\ ISchool of Physics and Astronomy, University of Manchester, Manchester, M13 9PL, UK \\ *D.Smith19@edu.salford.ac.uk; **J.E.Proctor@salford.ac.uk
}




\begin{abstract}
The chemical reaction between hydrogen and purely $\mathrm{sp}^{2}$-bonded graphene to form graphene's purely $\mathrm{sp}^{3}$-bonded analogue, graphane, potentially allows the synthesis of a much wider variety of novel two-dimensional materials by opening a pathway to the application of conventional chemistry methods in graphene. Graphene is currently hydrogenated by exposure to atomic hydrogen in a vacuum, but these methods have not yielded a complete conversion of graphene to graphane, even with graphene exposed to hydrogen on both sides of the lattice. By heating graphene in molecular hydrogen under compression to modest high pressure in a diamond anvil cell $(2.6-5.0 \mathrm{GPa})$, we are able to react graphene with hydrogen and propose a method whereby fully-hydrogenated graphane may be synthesized for the first time.
\end{abstract}

Keywords: graphene, functionalized graphene, hydrogenated graphene, graphane, diamond anvil cell

Graphane - the chemical derivative of graphene with purely $\mathrm{sp}^{3}$ bonding and a hydrogen atom bonded to each carbon (stoichiometry $\mathrm{CH}$ ) - has been the subject of theoretical studies ${ }^{1}$, but as yet has not been synthesized, with only partial hydrogenation of graphene most commonly achieved through exposure of graphene to atomized plasma in vacuum. ${ }^{2,3}$ The high surface area-to-volume ratio of graphene makes it a desirable material for applications from the nanoscale up to macroscopic materials, such as in biomedicine ${ }^{4}$, single-molecule gas sensing ${ }^{5}$ and novel composite materials ${ }^{6}$, were it to be suitably functionalized, but this first means overcoming the chemical inertness of graphene - it reacts only with hydrogen, ${ }^{2}$ oxygen ${ }^{7}$ and halogens. ${ }^{8,9}$ Oxygen's valency of 2 results in a tendency to bond to the graphene lattice on more than one carbon atom, causing damage to the material, and halogens form strong bonds with carbon that are not easily broken. ${ }^{8}$ 
Hydrogen, however, could easily be replaced with other functional groups, making hydrogenated graphene of interest as a starting point for graphene chemistry.

Chemical functionalization of graphene brings about changes in its electronic properties, with theory predicting that fully-hydrogenated graphene is an electronic insulator ${ }^{1}$ and early experiments on partially-hydrogenated graphene and chlorinated graphene revealing that even low levels of functionalization drastically reduce conductivity compared with pristine graphene. ${ }^{2,9,10}$ Hydrogenated graphene electronic devices are potentially possible by selectively removing areas of hydrogen to leave quantum wires (or nano-ribbons) and quantum dots of pristine graphene patterned into the material, with pristine graphene's electronic properties returning in those locations. ${ }^{11}$

The reaction between graphene and hydrogen is easily reversible - demonstrating the feasibility of carbon-based materials in solid-state hydrogen storage, where there is a need to reversibly bond hydrogen to the lightest element(s) possible to maximize hydrogen storage capacity by weight. A proper understanding of, and ability to control, the reaction between pristine graphene and hydrogen will therefore be critical in the development of carbon-based solid state hydrogen storage materials.

Decades prior to the discovery of graphene, high pressure and high temperature conditions were used in the conversion of bonding nature in carbon species from planar, graphitic $\mathrm{sp}^{2}$ into tetrahedral, diamond-like $\mathrm{sp}^{3}$ with the enormous heated presses employed by 20th century scientists in their successful first synthesis of man-made diamond from graphite seeds. ${ }^{12}$ More recently, $\mathrm{C}_{60}$ molecules and glassy carbon have been converted to nanocrystalline diamond under high pressure. ${ }^{13,14}$ By acknowledging these phenomena, it seems reasonable to expect that compressing graphene to high pressures under high temperature in the presence of hydrogen 
should facilitate the conversion of purely- $\mathrm{sp}^{2}$ bonded graphene into its $\mathrm{sp}^{3}$ bonded counterpart graphane, with hydrogen atoms terminating the $\mathrm{sp}^{3}$ bonds normal to the surface.

In the present work, we demonstrate use of high pressure and high temperature as a valid method of hydrogenating graphene. We confirm hydrogenation of graphene in the same fashion as other authors, by observing signature changes in the Raman spectra of samples upon reaction with hydrogen and by removing hydrogen from the samples to return to pristine graphene.

\section{Results and discussion}

Figure 1 shows the evolution of the Raman spectrum of graphene on copper foil after reaction with molecular hydrogen at $5.0 \mathrm{GPa}$ and $200^{\circ} \mathrm{C}$ in a diamond anvil cell. Samples of monolayer graphene were grown by chemical vapor deposition (CVD) onto substrates of copper foil. A small ( $\sim 200 \mu \mathrm{m}$ in diameter) piece of copper covered with graphene was cut and loaded into gasketed diamond anvil cells. The cells were equipped with pre-indented stainless steel gaskets alongside several pieces of ruby crystal to serve as a pressure calibrant. Hydrogen was loaded into the pressure chamber using high pressure gas loading at 2 kbar. Pressure was maintained at 5.0 GPa as temperature was raised to $200^{\circ} \mathrm{C}$, maintained for 15 minutes and steadily reduced to room temperature. Care was taken to ensure temperature inside the sample chamber had fallen below $50^{\circ} \mathrm{C}$ before pressure was relieved, as heating in the absence of pressure removes hydrogen from hydrogenated graphene as low as $75^{\circ} \mathrm{C} . .^{15}$ 

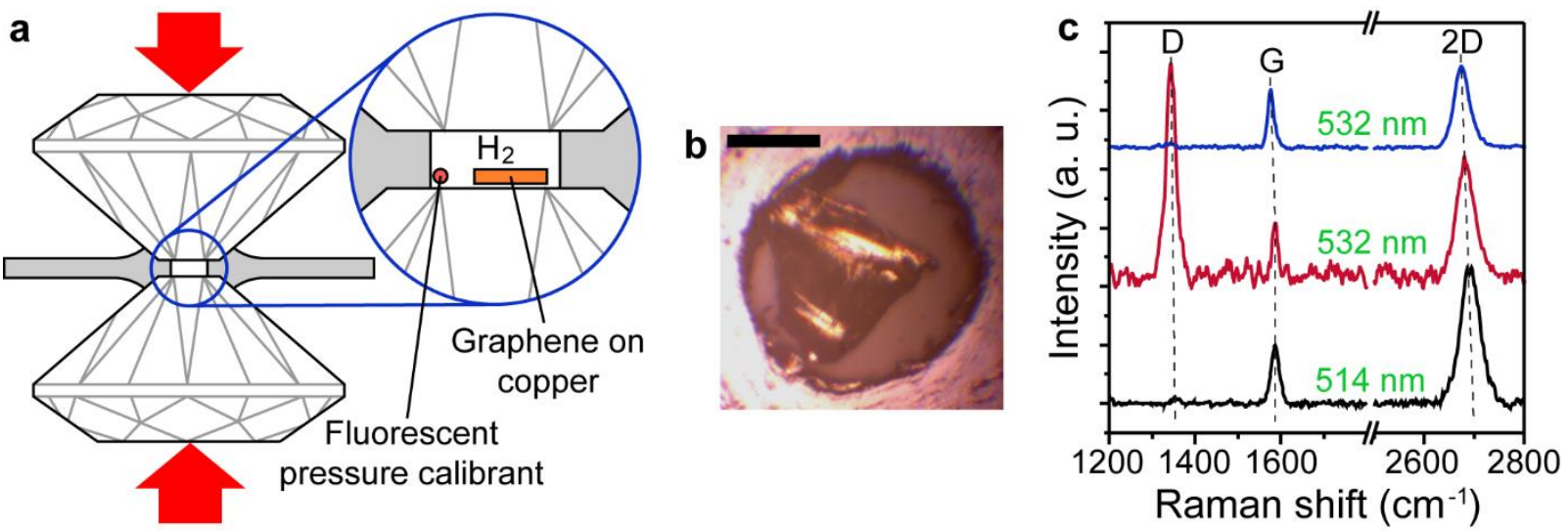

Figure 1. (a) Diamond anvil cell arrangement and contents of pressure chamber, red arrows indicate force applied to generate pressure. (b) Optical micrograph of sample of graphene on $\mathrm{Cu}$ substrate in an atmosphere of solid, molecular deuterium prior to heating - scale bar is $100 \mu \mathrm{m}$.

(c) Raman spectrum of graphene (black) prior to hydrogen treatment, (red) after treatment at 5.0 $\mathrm{GPa}$ and $200^{\circ} \mathrm{C}$ and (blue) after vacuum annealing at $200^{\circ} \mathrm{C}$ to remove hydrogen. Raman excitation wavelength is indicated next to spectra.

Pressure inside the pressure chamber of a diamond anvil cell is commonly measured using calibrated shifts in the positions of photoluminescence lines of various crystals or the shift in Raman peak position of the diamond anvil itself as pressure is increased. For pressure measurements at room temperature, we use the photoluminescence peaks of ruby crystal. ${ }^{16}$ For accurate measurements of pressure at high temperature, we refer to stress-induced shift of the diamond Raman peak at the point of contact between the culet and sample chamber, ${ }^{17}$ the shifting of photoluminescence peaks of samarium-doped yttrium aluminium garnet (Sm:YAG) crystal which exhibit immeasurably small temperature dependence up to $800 \mathrm{~K}^{18}$ or the ruby photoluminescence scale corrected or high temperature. ${ }^{19}$

After treatment in hydrogen at combined high pressure and high temperature, a sharp peak in the Raman spectrum of graphene appears at $1350 \mathrm{~cm}^{-1}-$ the D peak. This peak is forbidden in 
pristine graphene due to symmetry-based Raman selection rules, but appears due to the presence of defects or bonded atoms on the lattice that create $\mathrm{sp}^{3}$-like sites. ${ }^{20,21}$ It is important to note that the $\mathrm{D}$ peak and its derivative are a prominent feature in graphene suffering from disorder or damage $^{22}$ as well as chemically modified graphene. However, in the case of hydrogenated graphene, the hydrogen (and thus the D peak) can be removed by annealing to modest high temperatures, ${ }^{15}$ which we do not expect in the case of graphene with structural defects since graphitization of carbon materials (the structural annealing to form graphitic - i.e. $\mathrm{sp}^{2}-\mathrm{sheets}$ ) is not activated at temperatures below $500^{\circ} \mathrm{C} .^{23}$ The spectrum in Figure 1 of hydrogenated graphene annealed overnight at $200^{\circ} \mathrm{C}$ under ultra-high vacuum $\left(10^{-10} \mathrm{mbar}\right)$ thus provides evidence that graphene reacts with molecular hydrogen at $5.0 \mathrm{GPa}$ and $200^{\circ} \mathrm{C}$, and that these conditions do not simply structurally affect the graphene lattice.

The D peak also allows indirect estimation of the extent of hydrogenation by considering each hydrogen atom as an $\mathrm{sp}^{3}$ impurity on the lattice. The ratio $I_{D} / I_{G}$ between the intensity of the D peak, $I_{D}$, and of the $\mathrm{G}$ peak at $1580 \mathrm{~cm}^{-1}, I_{G}$, can be used to estimate the size of the $\mathrm{sp}^{2}$ crystallites separating these impurities. ${ }^{24} \mathrm{~A}$ benchmark of $I_{D} / I_{G}=2$ being equivalent to $\sim 10 \%$ atomic coverage of hydrogen is widely used in the literature, ${ }^{3,10}$ but with little justification. Raman spectroscopy would confirm the synthesis of fully-hydrogenated graphane, as the Raman scattering crosssection of $\mathrm{sp}^{3}$ bonded carbon is considerably smaller than for $\mathrm{sp}^{2}$ bonding, ${ }^{25}$ such that in the graphane limit the Raman signal from a monolayer would be immeasurable - as already reported for the full conversion of graphene's bonding nature via fluorination. ${ }^{8}$

In accordance with other authors, D peak intensity in our hydrogenated graphene varies across the sample. ${ }^{2}$ In its most intense location, the $I_{D} / I_{G}$ ratio for graphene hydrogenated by high pressure and high temperature in Figure 1 is 3.7, suggesting a level of hydrogenation that is competitive 
with current methods employing atomized hydrogen under low pressure to react with graphene. ${ }^{2,26,27}$

Figure 2a shows the evolution of the D peak in the Raman spectrum of graphene after reaction with molecular hydrogen for 1 hour at $200^{\circ} \mathrm{C}$ and $2.6 \mathrm{GPa}$ - according to the shift in ruby photoluminescence lines ${ }^{16}$ corrected for high temperatures. ${ }^{19}$ The $I_{D} / I_{G}$ ratio of 0.7 suggest a low level of hydrogenation. Figure $2 \mathrm{~b}$ shows the same evolution in $\mathrm{D}$ peak after 15 minutes of reaction between graphene and molecular hydrogen at $200^{\circ} \mathrm{C}$ and $4.0 \mathrm{GPa}$ - measured using shift in the Y2 line of Sm:YAG. ${ }^{21}$ A more intense D peak $\left(I_{D} / I_{G}=2.3\right)$ as well as the emergence of the D' peak is observed under these conditions. Although the reaction at 2.6 GPa was carried out over a much longer time period, there is a marked difference in the $\mathrm{D}$ peak intensity - and thus the hydrogenation levels - between those samples and the samples treated at 4.0 and especially 5.0 $\mathrm{GPa}$ at the same temperature, allowing us to infer that pressure has an influence on the reaction rate with hydrogen or on the maximum possible extent of the reaction.
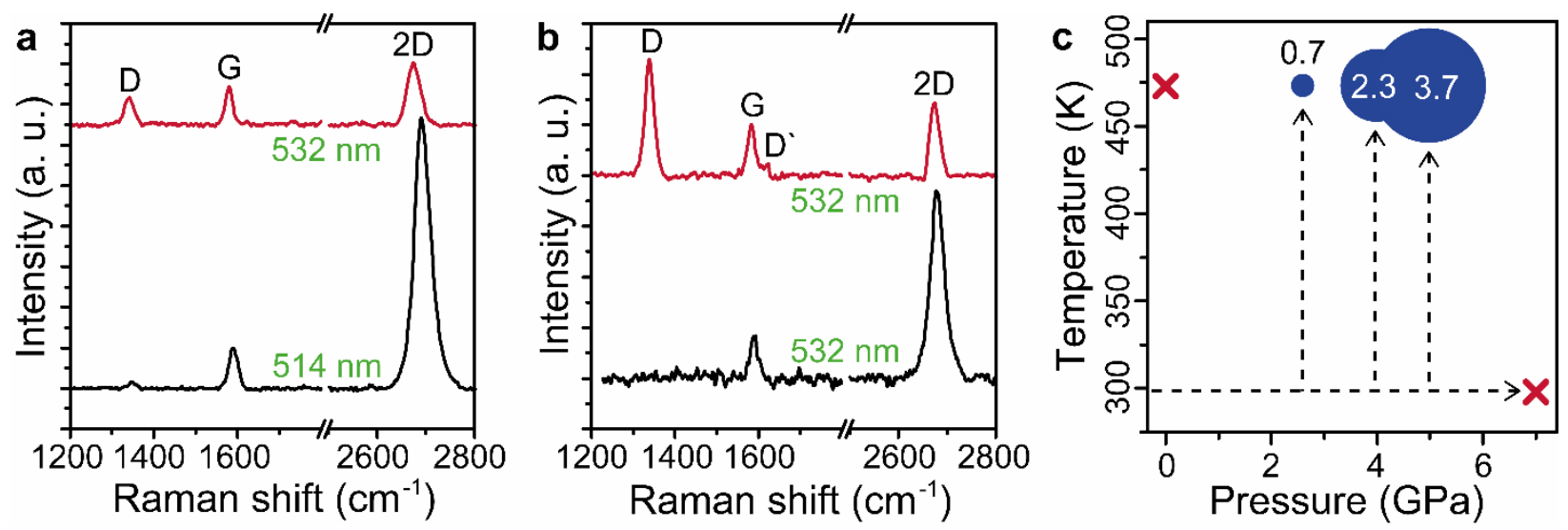

Figure 2. (a) Raman spectrum of (black) pristine graphene before and (red) hydrogenated graphene after treatment at $2.6 \mathrm{GPa}$ and $200^{\circ} \mathrm{C}$ (b) Raman spectrum of (black) pristine graphene before and (red) hydrogenated graphene after treatment at $4.0 \mathrm{GPa}$ and $200^{\circ} \mathrm{C}$. Raman excitation wavelength is indicated next to spectra. (c) P-T phase diagram of graphene-hydrogen reaction 
from the presented work, with blue bubbles indicating its extent and red crosses showing null conditions. Dashed arrows show the P-T paths taken in each experiment.

Samples of graphene have also been pressurized in hydrogen at ambient temperature, to $7.4 \mathrm{GPa}$. These samples were left in hydrogen at high pressure for 4 hours before pressure was released, and no measurable D peak activity was seen across the graphene sample - indicating a lack of reaction between the graphene and hydrogen. Graphene was also pressurized to $9.0 \mathrm{GPa}$ in molecular deuterium at room temperature, where pressure was maintained for 36 days. Again, when pressure was released there was no development of D peak in the observed Raman spectrum across the sample. The results of these room-temperature experiments suggest that graphene will not react with molecular hydrogen (or deuterium) at high pressure in the absence of high temperature, or that pressure much higher than $10 \mathrm{GPa}$ are necessary to facilitate the reaction - drastically reducing the possible sample size for an experiment. The range of reaction conditions applied allows the plotting of a P-T phase diagram of graphene-hydrogen reactions, displayed in Figure 2c, where we see hydrogenation of graphene activated above $2.6 \mathrm{GPa}$ and its increasing extent with pressure at $200^{\circ} \mathrm{C}$ and the absence of hydrogenation in graphene compressed at room temperature.

\section{Conclusion}

In summary, we have demonstrated that sufficient pressure and temperature in a diamond anvil cell will initiate the reaction between graphene and molecular hydrogen. The hydrogen coverage we estimate using the $I_{D} / I_{G}$ ratio of the hydrogenated graphene Raman spectrum is competitive with current methods employing atomic hydrogen plasma at low pressures and we have seen a strong dependence of the hydrogen content of our samples on the pressure of the reaction. By production of samples of monolayer graphene suspended over an aperture in a substrate, ${ }^{2}$ and by 
exposing graphene to higher pressure and temperature conditions for longer times, we propose that reaction at high pressure and high temperature could allow synthesis of fully-hydrogenated graphane for the first time.

\section{Experimental section}

Sample preparation. Graphene samples for all experiments were grown by CVD using decomposition of $\mathrm{CH}_{4}{ }^{28}$ onto large $(1 \mathrm{inch})$ sheets of $\mathrm{Cu}$ foil with a thickness of $8 \mu \mathrm{m}$, resulting in complete coverage of the $\mathrm{Cu}$ foil with a network of small (up to $500 \mu \mathrm{m}$ ) crystals of monolayer graphene on both sides of the foil. Samples for diamond anvil cell experiments were formed by cutting suitably small (typically $200 \mu \mathrm{m}$ ) sections of graphene-coated $\mathrm{Cu}$ substrate using a finepoint needle, and then verifying using Raman spectroscopy that no damage had occurred to the monolayer graphene.

Photoluminescence and Raman spectroscopy. Photoluminescence measurements on a ruby crystal in the sample chamber to determine pressure ${ }^{16}$ were performed using $532 \mathrm{~nm}$ solid state laser, photoluminescence measurements on Sm:YAG were performed with $405 \mathrm{~nm}$ solid state laser. ${ }^{18}$ Spectra during experiments were collected on either a Horiba iHR320 with Symphony CCD or Andor Shamrock-303i-A with iDUS CCD, utilizing a Mitutoyo 10x objective lens $(\mathrm{f}=$ $200 \mathrm{~mm}$ ). Raman spectroscopy on diamond anvils to determine pressure was performed using the Horiba spectrometer with a Nachet 20x objective lens $(\mathrm{f}=150 \mathrm{~mm})$ and $532 \mathrm{~nm}$ solid state laser. Raman spectroscopy of graphene samples was performed using a Renishaw Raman microscope with Leica 50x objective lens $(\mathrm{f}=0.75 \mathrm{~mm})$ and $514 \mathrm{~nm}$ solid state laser and Horiba spectrometer with Olympus Plan N 40x objective lens $(\mathrm{f}=0.65 \mathrm{~mm})$ and $532 \mathrm{~nm}$ solid state laser. Fitting of 
experimental data to find peak positions and relative intensities was performed using MagicPlot 2.5.1 software package after background subtraction in OriginPro 9.

Diamond anvil cell high pressure experiments. We used custom-constructed piston-cylinder diamond anvil cells. Diamonds with culets of 1,000, 600 and $450 \mu \mathrm{m}$ diameter were used depending on pressure requirements and stainless steel gaskets prepared by appropriately indenting and drilling $200 \mu \mathrm{m}$-thick stainless steel plates were used throughout. Hydrogen and deuterium were loaded at 2 kbar via the high pressure gas loading method ${ }^{29,30}$ to act as the pressure transmitting medium as well as a reagent. Temperature was applied by a $100 \mathrm{~V}$ Watlow nozzle heater placed around the exterior of the diamond anvil cell and controlled by a purpose-built circuit which uses feedback from a type $\mathrm{K}$ thermocouple inside the cell - close to the diamonds - to adjust the power supplied to the heater. In this way, stable $\left( \pm 10^{\circ} \mathrm{C}\right)$ temperatures can be maintained for extended periods.

Annealing of hydrogenated graphene. Annealing of samples of hydrogenated graphene was carried out in an ultra-high vacuum system ${ }^{31}$ pumped with ion pumps and titanium sublimation pumps to a base pressure of $3 \times 10^{-10}$ mbar. The sample was supported on a tantalum plate which was heated to $200^{\circ} \mathrm{C}$ using radiation from a tungsten filament mounted behind the sample plate and the temperature was monitored with a type $\mathrm{K}$ thermocouple.

\section{Author contributions \\ J.P. designed the project, conducted high pressure, high temperature experiments and assisted in writing the paper. D.S. prepared samples for experiments, conducted all high pressure, high temperature experiments, and wrote the paper. C.S. assisted with some high pressure, high}


temperature experiments. K.S.N. provided large-scale samples of graphene grown on copper substrates used throughout. R.T.H. and E.G. assisted with hydrogen loading in experiments. I.C. and M.P.H. assisted with Raman analysis of pristine and hydrogenated graphene samples. C.M. and V.V. annealed hydrogenated samples.

\section{Acknowledgements}

The presented work was supported by the Royal Society through the award of a Research Startup Grant (RG110437). High temperature diamond anvil cells were constructed by N. Parkin (University of Hull) and M. Clegg (University of Salford). The Raman spectrometer used for high pressure, high temperature experiments was constructed with the assistance of I. Morrison (University of Salford). D.S. would like to thank V. Markevich (University of Manchester) for efforts in annealing hydrogenated graphene. Sm:YAG crystals were grown and kindly provided by D. M. Trots, A. Kurnosov and D. J. Frost (University of Bayreuth).

\section{References}

1. Sofo, J.; Chaudhari, G.; Barber, G. Graphane: A Two-Dimensional Hydrocarbon. Phys. Rev. B 2007, 75, 153401.

2. Elias, D. C.; Nair, R. R.; Mohiuddin, T. M. G.; Morozov, S. V.; Blake, P.; Halsall, M. P.; Ferrari, A. C.; Boukhvalov, D. W.; Katsnelson, M. I.; Geim, A. K. et al. Control of Graphene's Properties by Reversible Hydrogenation: Evidence for Graphane. Science 2009, 323, 610-613.

3. Pumera, M.; Wong, C. Graphane and Hydrogenated Graphene. Chem. Soc. Rev. 2013, 42, 59875995. 
4. Yang, K.; Feng, L.; Shi, X; Liu, Z. Nano-Graphene in Biomedicine: Theranostic Applications. Chem. Soc. Rev. 2013, 42, 530-547.

5. Schedin, F.; Geim, A. K.; Morozov, S. V.; Hill, E. W.; Blake, P.; Katsnelson, M. I.; Novoselov, K. S. Detection of Individual Gas Molecules Adsorbed on Graphene. Nat. Mater. 2007, 6, 652655.

6. Stankovich, S.; Dikin, D. A.; Dommett, G. H. B.; Kohlhaas, K. M.; Zimney, E. J.; Stach, E. A.; Piner, R. D.; Nguyen, S. T.; Ruoff, R. S. Graphene-Based Composite Materials. Nature 2006, 442, 282-286.

7. Eda, G.; Chhowalla, M. Chemically Derived Graphene Oxide: Towards Large-Area Thin-Film Electronics and Optoelectronics. Adv. Mater. 2010, 22, 2392-2415.

8. Nair, R. R.; Ren, W.; Jalil, R.; Riaz, I.; Kravets, V. G.; Britnell, L.; Blake, P.; Schedin, F.; Mayorov, A. S.; Yuan, Shengjun et al. Fluorographene: A Two-Dimensional Counterpart of Teflon. Small 2010, 6, 2877-2884.

9. Li, B.; Zhou, L.; Wu, D.; Peng, H.; Yan, K.; Zhou, Y.; Liu, Z. Photochemical Chlorination of Graphene. ACS Nano 2011, 5, 5957-5961.

10. Burgess, J. S.; Matis, B. R.; Robinson, J. T.; Bulat, F. A.; Keith Perkins, F.; Houston, B. H.; Baldwin, J. W. Tuning the Electronic Properties of Graphene by Hydrogenation in a Plasma Enhanced Chemical Vapor Deposition Reactor. Carbon 2011, 49, 4420-4426.

11. Sessi, P., Guest, J. R., Bode, M.; Guisinger, N. P. Patterning Graphene at the Nanometer Scale via Hydrogen Desorption. Nano Lett. 2009, 9, 4343-4347. 
12. Bovenkerk, H. P.; Bundy, F. P.; Hall, H. T.; Strong, H. M.; Wentorf, R. H. Preparation of Diamond. Nature 1959, 184, 1094-1098.

13. Regueiro, M. N.; Monceau, P.; Hodeau, J.-L. Crushing C60 to Diamond at Room Temperature. Nature 1992, 355, 237-239.

14. Dubrovinsky, L.; Dubrovinskaia, N.; Prakapenka, V. B.; Abakumov, A. M. Implementation of Micro-Ball Nanodiamond Anvils for High-Pressure Studies Above 6 Mbar. Nat. Commun. 2012, $3,1163$.

15. Luo, Z.; Yu, T.; Kim, K.-J.; Ni, Z.; You, Y.; Lim, S.; Shen, Z.; Wang, S.; Lin, J. ThicknessDependent Reversible Hydrogenation of Graphene Layers. ACS Nano 2009, 3, 1781-1788.

16. Mao, H. K.; Xu, J.; Bell, P. M. Calibration of the Ruby Pressure Gauge to 800 Kbar under Quasi-Hydrostatic Conditions. J. Geophys. Res. 1986, 91, 4673-4676.

17. Akahama, Y.; Kawamura, H. Pressure Calibration of Diamond Anvil Raman Gauge To 310 Gpa. J. Appl. Phys. 2006, 100, 043516.

18. Goncharov, A. F.; Zaug, J. M.; Crowhurst, J. C.; Gregoryanz, E. Optical Calibration of Pressure Sensors for High Pressures and Temperatures. J. Appl. Phys. 2005, 97, 094917.

19. Rekhi, S.; Dubrovinsky, L; Saxena, S. Temperature-Induced Ruby Fluorescence Shifts up to a Pressure of 15 Gpa in an Externally Heated Diamond Anvil Cell. High Temp. High Pres. 1999, $31,299-305$.

20. Wang, Y., Alsmeyer, R.; McReery, R. L. Raman Spectroscopy of Carbon Materials: Structural Basis of Observed Spectra. Chem. Mater. 1990, 2, 557-563. 
21. Ferrari, A. C.; Basko, D. M. Raman Spectroscopy as a Versatile Tool for Studying the Properties of Graphene. Nat. Nanotechnol. 2013, 8, 235-246.

22. Lucchese, M. M.; Stavale, F.; Ferreira, E. H. M.; Vilani, C.; Moutinho, M. V. O.; Capaz, R. B.; Achete, C. A.; Jorio, A. Quantifying Ion-Induced Defects and Raman Relaxation Length in Graphene. Carbon 2010, 48, 1592-1597.

23. Saito, R.; Dresselhaus, G.; Dresselhaus, M. S. Physical Properties of Carbon Nanotubes; Imperial College Press: London, 2004.

24. Cançado, L. G.; Takai, K.; Enoki, T.; Endo, M.; Kim, Y. A.; Mizusaki, H.; Jorio, A.; Coelho, L. N.; Magalhães-Paniago, R.; Pimenta, M. A. General Equation for the Determination of the Crystallite Size L $\mathrm{a}_{\mathrm{a}}$ of Nanographite By Raman Spectroscopy, Appl. Phys. Lett. 2006, 88, 26-29.

25. Ferrari, A. C.; Robertson, J. Interpretation of Raman Spectra of Disordered and Amorphous Carbon. Phys. Rev. B 2000, 61, 14095-14107.

26. Ryu, S.; Han, M. Y.; Maultzsch, J.; Heinz, T. F.; Kim, P.; Steigerwald, M. L.; Brus, L. E. Reversible Basal Plane Hydrogenation of Graphene. Nano Lett. 2008, 8, 4597-4602.

27. Matis, B. R.; Burgess, J. S.; Bulat, F. A.; Friedman, A. L.; Houston, B. H.; Baldwin, J. W. Surface Doping and Band Gap Tunability in Hydrogenated Graphene. ACS Nano 2012, 6, 17-22. 28. Li, X.; Cai, W.; An, J.; Kim, S.; Nah, J.; Yang, D.; Piner, R.; Velamakanni, A.; Jung, I.; Tutuc, E. et al. Large-Area Synthesis of High-Quality and Uniform Graphene Films on Copper Foils. Science 2009, 324, 1312-1314. 
29. Howie, R. T.; Guillaume, C. L.; Scheler, T.; Goncharov, A. F.; Gregoryanz, E. Mixed Molecular and Atomic Phase of Dense Hydrogen. Phys. Rev. Lett. 2012, 108, 1-5.

30. Howie, R. T.; Dalladay-Simpson, P; Gregoryanz, E. Raman Spectroscopy of Hot Hydrogen Above 200 GPa. Nat. Mater. 2009, 14, 495-499.

31. Zan, R.; Muryn, C.; Bangert, U.; Mattocks, P.; Wincott, P.; Vaughan, D.; Li, X.; Colombo, L.; Ruoff, R. S.; Hamilton, B. et al. Scanning Tunnelling Microscopy of Suspended Graphene. Nanoscale 2012, 4, 3065-3068. 

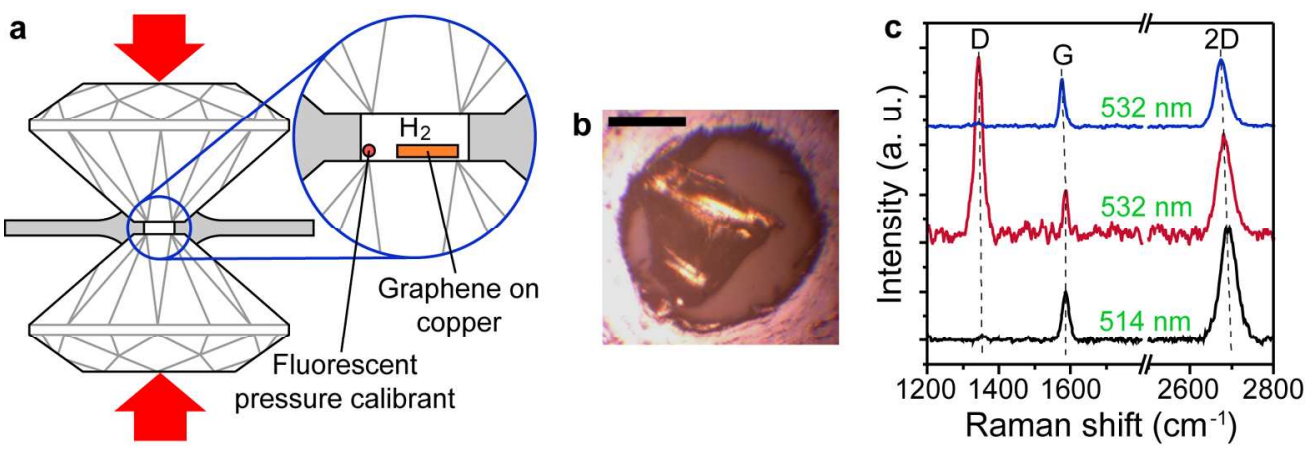

Figure 1. (a) Diamond anvil cell arrangement and contents of pressure chamber, red arrows indicate force applied to generate pressure. (b) Optical micrograph of sample of graphene on Cu substrate in an atmosphere of solid, molecular deuterium prior to heating - scale bar is $100 \mu \mathrm{m}$. (c) Raman spectrum of graphene (black) prior to hydrogen treatment, (red) after treatment at $5.0 \mathrm{GPa}$ and $200^{\circ} \mathrm{C}$ and (blue) after vacuum annealing at $200^{\circ} \mathrm{C}$ to remove hydrogen. Raman excitation wavelength is indicated next to spectra. $165 \times 55 \mathrm{~mm}(300 \times 300 \mathrm{DPI})$ 

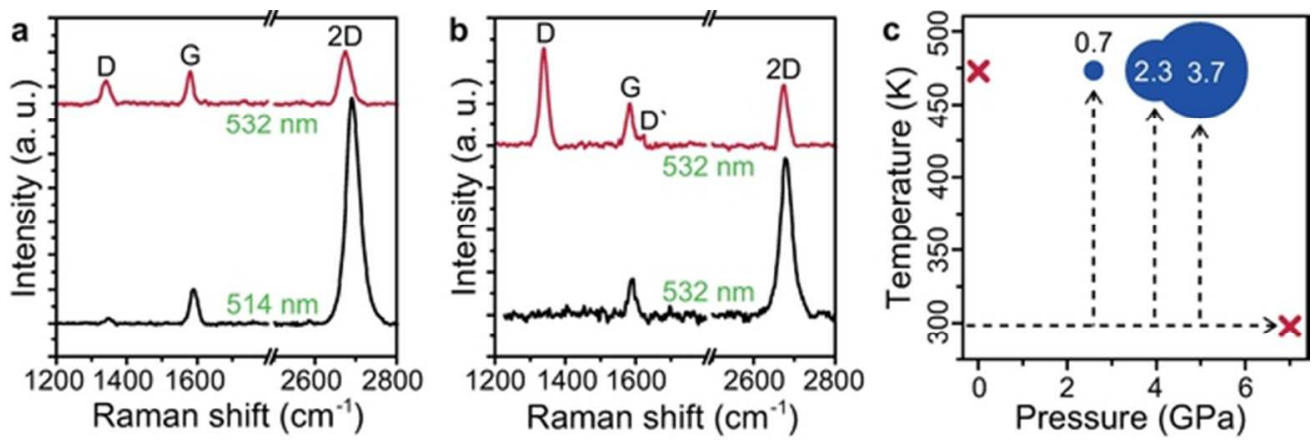

Figure 2. (a) Raman spectrum of (black) pristine graphene before and (red) hydrogenated graphene after treatment at $2.6 \mathrm{GPa}$ and $200^{\circ} \mathrm{C}$ (b) Raman spectrum of (black) pristine graphene before and (red) hydrogenated graphene after treatment at $4.0 \mathrm{GPa}$ and $200^{\circ} \mathrm{C}$. Raman excitation wavelength is indicated next to spectra. (c) P-T phase diagram of graphene-hydrogen reaction from the presented work, with blue bubbles indicating its extent and red crosses showing null conditions. Dashed arrows show the P-T paths taken in each experiment. $54 \times 18 \mathrm{~mm}(300 \times 300$ DPI $)$ 

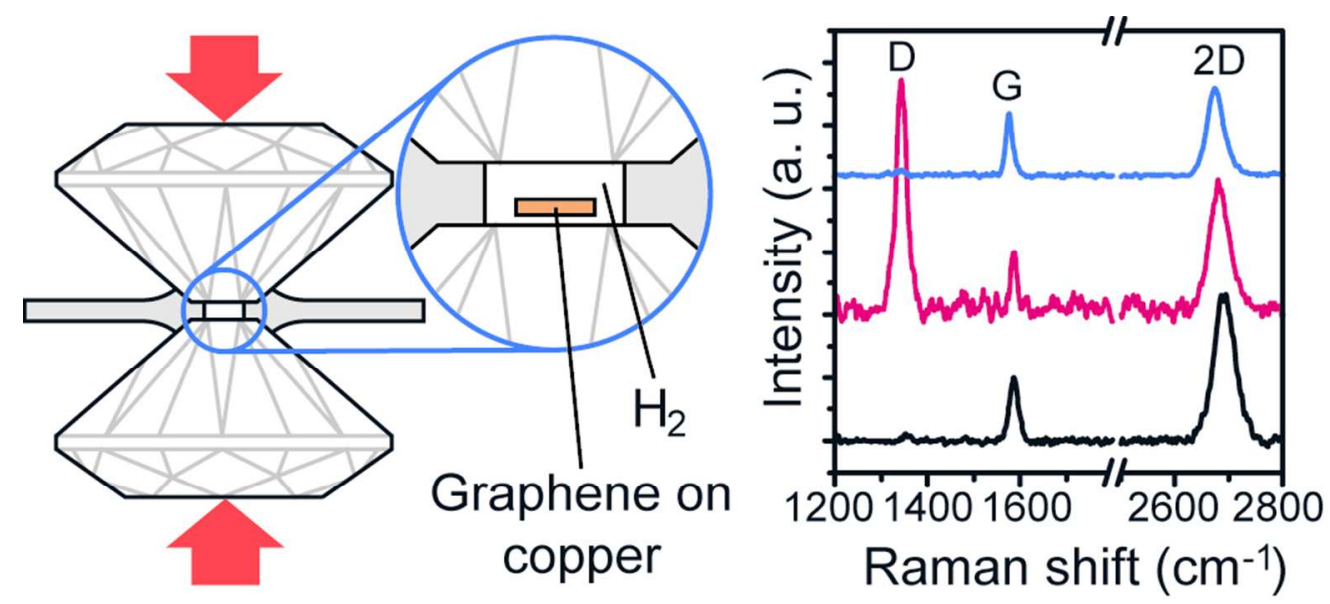

TOC graphic.

$88 \times 39 \mathrm{~mm}(300 \times 300 \mathrm{DPI})$ 